\title{
Non-synonymous Coding SNP
}

National Cancer Institute

\section{Source}

National Cancer Institute. Non-synonymous Coding SNP. NCI Thesaurus. Code C45397.

A Non-synonymous Coding SNP consists of a variation at an appreciable frequency between individuals of a single interbreeding population of a single nucleotide, due to base substitution, at an equivalent location within a protein-coding region of a gene that causes an alteration of the translation of the affected codon into a different amino acid in the protein product. A missense non-synonymous SNP results in an amino acid change in the protein product due to a codon change, while a nonsense non-synonymous SNP results in a termination codon. 